\title{
Le Mythe du feu et le principe féminin dans «Paysages» et Les Marches de sable d'Andrée Chedid
}

\author{
Judy Cochran \\ Denison Unioersity (Granoille, Ohio)
}

« aysages,» poème liminaire de Textes pour une figure, premier recueil en langue française d'André Chedid, est aussi un texte séminal annonçant des mythes fondamentaux de l'univers poétique et romanesque de cet auteur. ${ }^{1}$ Le poème signale d'abord l'aspect primordial du paysage désertique par la personnification des éléments qui le composent; ensuite il introduit la thématique de la recherche de soi à travers cette terre à la fois réelle et remémorée. L'oeuvre de Chedid témoigne de l'enfance de l'auteur en Egypte et sert d'arrière-fond au drame du «je» qui se découvre et s'affirme par rapport à la violence et au chaos des éléments premiers. ${ }^{2}$ Peu à peu, le paysage dévoile au poète son moi profond, dépouillé du masque social, et ce retour au terrain fondamental de l'inconscient lui restaure son regard premier. ${ }^{3}$ Le regard dénudé du poète lui permettra de pénétrer la réalité du paysage et de parvenir à l'expression authentique des images psychiques qui s'y trouvent reflétées. Jacques Izoard souligne ce lien entre Andrée Chedid et le paysage qu'elle dépeint: «Elle garde de son enfance égyptienne le goût de la beauté crue, sans faux miroirs» (39). La parole ainsi conquise amenera l'ouverture à autrui. Lieu de la découverte de soi, le paysage devient le lieu de la rencontre de l'autre, car le poete a pour but ultime la communication par la parole poétique. ${ }^{4}$

Au départ, le paysage chedidien se montre hostile aux humains et semble n'exister que pour mesurer l'une contre l'autre les forces destructrices du feu et de l'eau, ces deux éléments à la fois opposés et complémentaires.Le feu, personnifié par le Désert, est le premier élément qui domine le paysage liminaire. La femme est d'abord absente de ce lieu camusien-aride, implacable, à peine tempéré par le rare passage des hommes ou de l'oiseau. Par ailleurs, c'est l'eau, élément féminin par excellence, personnifiée par la Mer, qui triomphe des humains. L'influence féminine s'adoucit et se montre nourrissante et guérisseuse, s'infiltrant dans le paysage, grâce à l'arbre et à l'eau, «Que l'on 
nomme Oasis / Du nom d'une femme aimée.» La présence guérisseuse de la femme se fait sentir ici et prefigure son rôle ailleurs dans l'univers de Chedid où l'amour s'offre comme seule réponse possible à la violence primordiale du feu et de l'eau. Les qualités attribuées par Chedid au poète, quoique celui-ci paraisse comme un personnage masculin dans son univers, nous permettra d'aborder l'étude des Marches de sable où trois destins féminins s'accomplissent à travers les éléments du paysage désertique.

Plusieurs poèmes rattachent le poète à la symbolique du feu en reprenant les mythes d'Icare et de Prométhée. Icare, le protagoniste de «Seconde vie: Icare» est partagé entre le «cri» du soleil en lui-même et les voix quotidiennes qui lui représentent son devoir conjugal et filial au détriment de ses rêves d'absolu (Textes pour un poème, 83-88). Dans l'âme éprise d'absolu, le désir d'aller toujours plus en avant prend le dessus, et Icare suit l'appel du feu jusqu'au royaume de l'imaginaire où «Rêve et sang ne feront qu'un.» A l'instar de Bachelard, «l'appel du feu» conçu par Chedid représente le défi de l'individu contre les interdictions sociales, défi essentiel au devenir humain ( $L a P$ sychanalyse, 25-26). Chedid évoque le feu en faisant allusion au mythe de Prométhée dans d'autres poèmes qui, tout en dépeignant la fonction du poete comme voleur du feu, insistent davantage sur son rôle de bienfaiteur de l'humanité, ainsi que l'illustre l'invocation suivante: «Poète / Tu voles le vent pour nos faces / Le clair pour nos yeux / Le sel pour nos lèvres / Tu risques l'étoile / Tu cries notre raison» («Salut au poète,» Textes pour un poème: 107-108). «Blessures du poète» révèle la capacité régénératrice du poète, le rapprochant du phénix «toujours restauré,» «toujours renaissant» de ses cendres, tout en faisant valoir ses pouvoirs alchimiques de transformation et de fusion. Le poète recrée donc l'unité fondamentale de l'univers, résolvant en lui-même le feu et «le flot du dedans,» une des expressions par lesquelles Chedid désigne l'inconscient. («Blessures du poète,» Fraternité de la parole: $54-55$ ). Symboles à la fois de l'illimité et de l'abri, l'eau et le feu reflètent l'ambiguïté de la condition humaine que le poète parvient à réconcilier grâce à la parole.

L'exemple des trois héroïnes des Marches de sable nous servira à dégager le lien suggéré par ces poèmes entre personnages et paysage. Dans ce roman situé en Egypte au IVe siècle après Jésus-Christ, trois femmes d'âges et de conditions différentes, réfugiées au désert, se trouvent réunies par le hasard et poursuivent ensemble pendant un certain temps un destin commun qui les aidera à réaliser leurs destins individuels. L'auteur explique son choix du désert, symbolisé par le sablier qui évoque à la fois le temps qui coule et le temps arrêté. A son avis, «la terre dépouillée» du désert mène à la découverte de «ce qu'on porte d'essentiel en soi» (Accad,164). Pour deux de ces femmes, le désert ne sera qu'un passage vers une plus haute réalisation de leur vie humaine. L'une d'entre elles, manifestant ce que Bacheland nomme le «complexe de Prométhée," trouvera dans le feu du désert l'unique moyen d'apaiser la soif d'absolu qui la consume (La Psychanalyse,19).

Chez celle-ci, courtisane d'Alexandrie belle et instruite, «le feu sexualisé» se montre d'abord en tant que désir d'individualité ( $L a$ Psychanalyse,40). Cependant, à l'âge de vingt-cinq ans, ce personnage, appelé Marie, nom qui reflète son caractère paradoxal 
(vierge/Madeleine), s'enfuit au désert pour y vivre en anachorète. Dans ce lieu dépouillé, Marie, la courtisane, ressent le remords de ce qu'elle voit comme la perte de son humanité. Elle est hantée par «l'image de son jeune corps sacrifié» (30) et surtout par son souvenir de Jonahan [sic], l'homme qu'elle aurait pu aimer: «Pourquoi avoir sacrifié cet amour fragile au seul amour qui résistât à la durée? A cet absolu qui n'est peut-être qu'invention de l'esprit, qu'image sans empreinte?» (47). Marie esperre trouver dans le renoncement une réponse à «l'appel du feu» qui la tourmente (88). Elle donne son corps au soleil et se laisse consumer jusqu'à perdre son aspect humain. Finalement, la pulpe de sa chair semble se transformer en matière plus profonde, créant à l'intérieur de son corps desséché un paysage fertile, la justification de sa foi: «venue des tréfonds de la vie, et de ses propres entrailles, une terre féconde faisait surface» (48). Elle découvre par moments au fond d'elle-même l'illimité qu'elle cherchait, et que Thémis, le narrateur, reconnaît comme la source del'imaginaire: «terreau commun dans lequel les inventeurs, les bâtisseurs, les créateurs de mots, de sons, d'images ne cessent de puiser; humus d'où les fous d'absolu, d'indicible, cherchent contre tout argument à tirer réponses, et qui secrete une part du fertile univers» (48). Ici, la terre prend une valeur régénératrice propre à la femme de même qu'à l'artiste. Marie se rapproche encore du personnage du poète par la capacité alchimique de l'imagination que Bachelard qualifie de "principe de semence» ( $L a$ Psychanalyse,85). De même, dans le lexique d'Andrée Chedid la semence, présente chez la femme aussi bien que chez l'homme, est synonyme de l'imaginaire dont la parole est l'expression ultime. Après avoir passé neuf ans au désert, Marie trouve en la parole son unique soutien: elle parle aux étoiles et au vent, écrit sur les murs de sa caverne et sur l'écorce des arbres. Cependant, elle finit par préférer les images «sans empreinte,» et par effacer ses paroles qu'elle trouve vaines, pour ne plus dessiner que sur le sable (49).

C'est à ce moment de l'histoire qu'elle rencontre Cyre dont le nom rappelle la source à l'origine de Cyrène, lieu du temple d'Apollon, ce dieu protecteur des arts. Orpheline de douze ans, qui a abandonné le couvent où on la maltraitait, l'enfant avait fait voeu de silence par reconnaissance envers un vieil ermite qui l'avait protégée, et elle ne parle plus que par le chant. Pour Cyre, le désert n'est qu'un passage qui mène vers l'oasis, "un endroit peuplé d'arbres et d'oiseaux» (150). Thémis, lorsqu'il rédige l'histoire des trois femmes, exprime ainsi la pensee de la jeune paysanne: «Ce qu'elle cherche, sans trop le savoir, c'est un lieu que n'encerclera aucun mur; un endroit, adouci par un arbre qui s'élèverait miraculeusement au milieu des sables» (14). L'arbre dans l'univers chedidien contient la sève et symbolise la vitalité aussi bien que l'abri. Dans le discours de Mace, l'ermite qui habite l'ancienne forteresse où les trois femmes trouvent asile, l'arbre devient une métaphore de la vie humaine: «pour s'accomplir, une vie humaine doit tendre à la fois vers ciel et terre; comme l'arbre, elle doit s'élancer vers le haut tout en répandant, en déployant ses racines dans ce sol qui le nourribs (166). Cyre ressemble à l'arbre qui lie la terre aux cieux et qui héberge l'oiseau. Comme l'oiseau, elle s'exprime par le chant et sa fuite se transforme en envol vers la liberté spirituelle, symbolisée par l'air. Au moment de quitter son couvent, tous les passereaux captifs dans sa poitrine se 
sont éveillés en battant des ailes (17). Leur envol correspond au sien. L'auteur explique l'évasion de l'enfant comme une marche «vers le chant» (Accad,165). Sa joie de vivre et la pureté de son chant fait d'elle une créature et de la terre et de l'air. Elle ne s'use pas au désert; elle y est de passage.

La simplicité de Cyre fait renaître en Marie le désir de la parole, mettant en cause la raison de sa fuite au désert. Pourtant, à force de dire sa vie, d'abord à l'enfant, ensuite à Athanasia, leur troisième compagne, et enfin à Thémis, Marie parvient à se comprendre. Il lui aura fallu ressentir la chaleur de l'amitie, aussi bien que les restes de la passion au cours d'une nuit passée avec Thémis, pour renoncer définitivement à ses attaches terrestres. Ce bref retour à l'amour charnel, tel que le raconte Thémis, se transforme plutôt en une expérience de la compassion: «Etendu et prenant contre moi cette femme, dont je voyais le regard s'animer et retrouver peu à peu son eau, je la berçais, je caressais sans dégoût sa peau calleuse, ses seins asséchés.... Ce que nous partagions, en cette nuit étrange, n'était pas de l'amour; et cependant j'ai chéri cette femme; de toutes mes forces je l'ai chérie, peut-être bien au-delà de l'amour!» (142)..$^{5}$ En Marie, la manifestation sexuelle du feu s'est consumée et purifiée: le feu devient lumière. L'eau qui remonte dans son regard marque sa renaissance par la compassion, essentielle à la réalisation de soi, et signifiée dans l'élaboration de la fonction du poète par la métaphore de «la traversée sensible» («La traversée sensible,» Textes pour un poème: 112). Cette renaissance de l'esprit à travers la chair imbue ce personnage des qualités du poète et représente la purification du feu sexualisé, car Marie, devenue anachorète véritable, ne souhaite plus être qu'esprit pur: «Alors, mêlée à l'air et à l'éternité, [son] âme deviendra le lieu du Seigneur» (230). Illuminée par le feu, elle comprend qu'il lui faudra vivre non seulement au désert, mais «du désert, » faisant partie de son anonymat total (231). ${ }^{6}$ Marie rend donc a Cyre la parole. En prenant sur elle le silence de Cyre, qui se meurt d'une piqûre de scorpion, Marie permet à celle-ci de prononcer les noms de ceux qu'elle chérit avant de les quitter, lui conférant brièvement le rôle du poète. Pendant qu'on emporte le corps de l'enfant pour l'enterrer dans l'île de ses rêves, Marie se prépare à franchir la dernière étape de son passage vers l'absolu: «En plus du silence, il lui reste à abolir au fond d'elle-même la source de la parole. Il faut cesser d'aimer, de souffrir, de sentir, il lui faut cesser d'être pour devenir l'espace et le lieu du Seigneur» (239). Consumée par la soif de l'absolu, Marie finit par s'unir à l'espace qui l'entoure. Trois ans après, Thémis imagine sa métamorphose complète, «détournée de l'humus de la parole et de la chair»: «Marie, squelette blanc, aussi épurée que le déserb» (246). ${ }^{7}$ Ainsi, s'effacent et se perpétuent ses «marches de sable,» car elle appartiendra désormais au royaume de l'air et de la lumière.

Des trois héroïnes, c'est Athanasia dont le nom signifie la négation de la mort, qui incarne le plus completement le principe féminin, car c'est elle qui réalise au plus haut degré les exigences de l'amour humain. Pacifiste et sceptique, Athanasia se trouve victime indirecte de la violence des combats politiques et religieux qui l'entourent et qui lui ont ôté enfants et époux. Comme Marie, elle se donne à l'érosion du désert, perdant son aspect de femme et devenant méconnaissable sauf pour Thémis, qui l'a 
toujours aimée d'un amour inavoué. Cette femme, qui vit pour et par l'amour des autres, offre à Cyre l'abri qu'elle cherchait. Elle devient la mere que l'enfant avait perdue: «une mere jamais vue; mais tellement désirée, tellement imaginée; une mère qui aurait vieilli, une mère puissante et secourable qui repousse menaces et moqueries, qui éloigne les dangers» (150). A son tour, c'est Cyre qui rachète le désert pour Athanasia. Tout en lui rappelant son fils mort aux mains des fanatiques, l'enfant la réconforte, la transporte loin des lieux arides, tel qu'elle lui explique: «Quand tu es là, le désert n'est plus du désert. Le désert devient une grande plage qui descend vers la mer» (154).

A l'encontre de Marie, Athanasia n'accepte pas Yanéantissement du désert, ce lieu de l'absence et du silence. Chez elle, l'amour se voit miroiter plutôt dans l'immensité de la mer. Tandis que Marie se voue à l'absolu, Athanasia lui reproche le repos qu'elle cherche au détriment de la vie. A la différence de celle-là, Athanasia embrasse le flux et l'éphémère de la condition humaine: «Ceux que l'on aime, ceux qui vous aiment font la vie. Le grouillement du monde fait la vie, tous les hommes avec leurs lambeaux de bonheur et de souffrance. La vie remue, change, broie, apaise, soulève. Cette immobilité n'est pas la vie» (157). D'ailleurs, laquelle des trois est mieux placée pour comprendre l'éternel «pourquoi» de l'humaine condition? Athanasia s'était réfugiée au désert à l'insu d'Andros, son mari, qui lui-même s'y était enfui pour sauvegarder leur fils aîné après le meurtre de leur cadet aux mains des fanatiques. Au bout d'une absence de sept ans, Athanasia retrouve son époux qui la prend pour un moine errant. Cinq ans passeront sans qu'Andros reconnaisse sa femme. Celle-ci dévoilera son identité à son époux sur son lit de mort pour entendre de lui le seul mot: «Pourquoi?» Athanasia se détourne de Dieu; elle rejette l'absolu que poursuivait son mari, et Thémis nous dit d'elle: «Ce 'pourquoi?' la poursuivra jusqu'à sa fin» (74). C'est le personnage le plus émouvant de l'histoire, à qui l'amour humain permet de surmonter sa douleur et de faire croître la vie autour de lui. Athanasia incarne l'Oasis du premier paysage dépeint par Chedid; elle porte en elle la force guérisseuse de l'amour. On la voit toujours entourée d'enfants, et à la fin du livre, on la verra revenir vers l'amour de Thémis.

Leur passage à travers le désert dévoile à ces trois femmes la valeur de l'amitié, qui transcende les barrières de caste et leur révèle leur propre identité. Réunies toutes trois dans ce lieu dépouillé, elles y trouvent un abri. De même, "Après le jardin,» un des premiers poèmes de Chedid, évoque l'aspect maternel du jardin terrestre, désigné comme le "ventre de la demeure» (Visage premier: 126). L'ancienne forteresse vers laquelle les trois hérö̈nes se dirigent devient cet asile favorable à l'échange fraternel qui mène à la connaissance de soi. En les protégeant contre le monde extérieur, ce lieu propre à la naissance spirituelle prend valeur de mère: «Comme une membrane protectrice, la roche les enveloppe toutes les trois; cet abri est un ventre où leurs liens se fortifient, où le coeur reprend souffle» (148). Le désert, capable d'anéantir la vie possède aussi la force de la restaurer: «terre dénudée où chaque parole, chaque regard, chaque geste prend sa véritable mesure, multiplie l'échange, tisse une peau neuve par-dessus les plaies: voila ce désert!» (148). Ainsi que le suggère le poème «Paysages»» 
la femme triomphe de l'aridité du désert, lui prêtant ses propres forces de réconfort et de régénération, faisant du désert un lieu d'espoir et d'amour.

\section{NOTES}

${ }^{1}$ Ce texte paraît dans Textes pour un poème, une réédition des poèmes écrits par Chedid entre 1949 et 1970 (17-19). Je l'ai cité en entier dans un article «Double-pays: l'univers poétique d'Andrée Chedid» à paraître dans le numéro d'automne 1994 de la Reoue Francophone de Louisiane.

${ }^{2}$ Dans L'Eau et les rêves, Gaston Bachelard a signalé l'importance du pays natal pour la matérialisation des images de l'inconscient (11). L'oeuvre d'André Chedid s'oriente autour de la métaphore du «double-pays» qui désigne la correspondance qu'elle ressent entre la terre et sa reflexion dans le domaine de l'imagination.

${ }^{3}$ Voir l'opposition masque/vérité traitée par Michael Bishop dans son article «Paysages.» Voir aussi l'entretien avec André Chedid cité par Jean Roger dans son commentaire sur «La Femme des longues patiences.»

'Toute l'oeuvre d'Andrée Chedid tend vers cette fin, telle que suggérée par. le titre Fraternité de la parole, recueil poétique qui a obtenu pour son auteur le plus haut prix de l'Académie Mallarmé en 1976.

${ }^{5}$ Le rôle de Thémis est assez complexe, car celui-ci sert en quelque sorte de porte-parole de l'auteur en même temps qu'il participe aux qualités du poète métaphorique qui restitue aux humains leur spiritualité. Dans une étude très sensible intitulée «L'Ecriture du désert dans Les Marches de sable d'A. Chedid," Bruno Tritsmans développe la part que Thémis contribue à dévoiler aux trois héroïnes la vérité de leurs vies en faisant de chacune d'elles objet de narration (112).

${ }^{6}$ Tritsmans souligne ailleurs ce qui sépare Marie des deux autres personnages féminins du récit. Tandis que Cyre et Athanasia s'attachent toujours à la terre et aux humains, Marie renonce complètement à la matière, s'identifiant totalement au lieu dépouillé qu'est le désert (112).

7Dans son étude «Un poème d'Andrée Chedid: Solfège du squelette,» Maurice Delacroix développe de façon très intéressante la signification des termes de la série musicale par rapport à l'image du squelette. Son rapprochement de la clef de sol de «Solfege du squelette» a la «clef de solitude» du poète est tout à fait apte à l'anachorète qui se donne à l'absence plutôt qu'à la présence, au silence plutôt qu'au chant (260-261). 


\section{BIBLIOGRAPHIE}

Accad, Evelyne. «Entretien avec Andrée Chedid.» Présence Francophone 24 (1982): 15774.

Bachelard, Gaston. L'Eau et les rêoes. Paris: José Corti, 1942. . La Psychanalyse du feu. Paris: Gallimard, 1949.

Bishop, Michael. «Paysages.» SUD (1991): 77-82.

Chedid, Andrée. Epreuves du vioant. Paris: Flammarion, 1983. . Fraternité de la parole. Paris: Flammarion, 1976. . Les Marches de sable. Paris: Flammarion, 1981. . Textes pour un poème. Paris: Flammarion, 1987. . Visage premier. Paris: Flammarion, 1972.

Delacroix, Maurice. «Un poème d'Andrée Chedid: Solfège du squelette.» Travaux de littérature 1 (1988): 253-263.

Izoard, Jacques. Andrée Chedid. Paris: Seghers, 1977.

Roger, Jean. «La Femme des longues patiences.» L'Hexagone 3 (1982): 278-283.

Tritsmans, Bruno. «L'Ecriture du désert dans Les Marches de sable d'A. Chedid.» Les Lettres romanes 45.1-2 (février/mai 1991): 109-115. 